\section{CONF-970847-3 \\ 3D ELECTROMAGNETIC MODELING USING \\ STAGGERED FINITE DIFFERENCES}

\author{
Gregory A. Newman and David L. Alumbaugh \\ Sandia National Laboratories \\ P.O. Box 5800, Albuquerque NM 87185-0750 \\ Tel: (505) 844-8158; Fax (505) 844-7354
}

Email: ganewma@sandia.gov

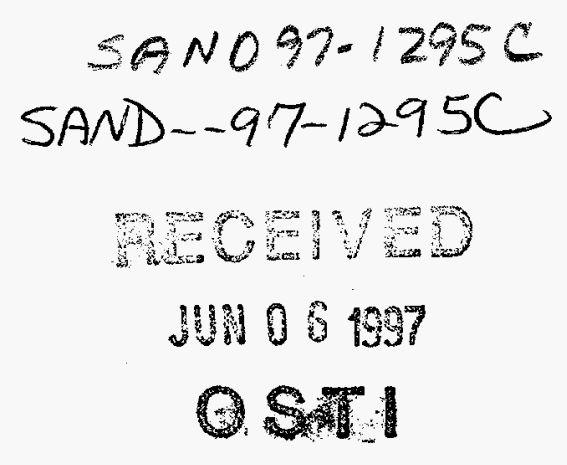

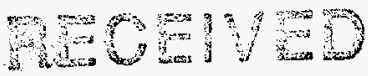

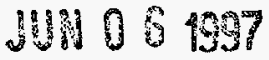

(1)

\begin{abstract}
The method of finite differences has been employed to solve a variety of 3D electromagnetic (EM) forward problems arising in geophysical applications. Specific sources considered include dipolar and magnetotelluric (MT) field excitation in the frequency domain. In the forward problem, the EM fields are simulated using a vector Helmholtz equation for the electric field, which are approximated using finite differences on a staggered grid. To obtain the fields, a complex-symmetric matrix system of equations is assembled and iteratively solved using the quasiminimum method (QMR) method. Perfectly matched layer (PML) absorbing boundary conditions are included in the solution and are necessary to accurately simulate fields in propagation regime (frequencies $>10 \mathrm{MHz}$ ). For frequencies approaching the static limit $(<10 \mathrm{KHz})$, the solution also includes a static-divergence correction, which is necessary to accurately simulate MT source fields and can be used to accelerate convergence for the dipolar source problem.
\end{abstract}

\section{INTRODUCTION}

For too long the interpretation of frequencydomain electromagnetic data arising from complex geologic media has been limited by the lack of interpretation tools. It is only within the last few years that the ability to model and invert complex 3D EM data is emerging. A key reason for this development has been the application of efficient finite-difference methods to the forward modeling problem.

Over the last five years, we have developed solutions to the 3D EM forward problem for frequencydomain applications ([1] and [2]). Progress has proceeded on several fronts, including the development of a fast 3D finite difference modeling code for both dipolar and MT or natural source (plane wave) fields. Key features of the forward modeling code is its ability to simulate fields from the propagation to diffusion regime (radar to inductive EM), the inclusion of the perfectly matched layer absorbing boundary conditions necessary to reduce the size of the model domain and accurately simulate wave propagation, and the ability to model 3D variations in electrical conductivity, dielectric permittivity and magnetic permeability. The modeling code also includes a static-divergence correction, which is necessary to insure accurate MT results. In this talk, we will review our efforts in forward modeling.

\section{THE FORWARD PROBLEM}

Theory

Assuming a time harmonic dependence of $e^{i a t}$ where $i=\sqrt{ }-1$, the vector Helmholtz equation for the electric field given by [1] is written here as

$$
\nabla_{\mathrm{e}} \times \mu_{\mathrm{o}} / \mu \nabla_{\mathrm{e}} \mathbf{x} \mathbf{E}+\omega \mu_{\mathrm{o}}(\sigma+i \omega \varepsilon) \mathbf{E}=\mathbf{S}
$$

In this expression the electrical conductivity, magnetic permeability and dielectric permittivity are denoted by $\sigma$, $\mu$ and $\varepsilon$ respectively, where $\mu_{0}$ is the magnetic permeability of free space. Specification of the source vector $S$, which includes the appropriate boundary conditions, determines whether dipolar or natural source (MT) field excitation is to be simulated. For dipolar source fields we have, for a total-field formulation

$$
\mathbf{S}=-i \omega \mu_{\mathrm{o}} \mathbf{J}_{\mathbf{p}}-i \omega \nabla_{\mathrm{h}} \times\left(\mu_{\mathrm{o}} / \mu \mathbf{M}_{\mathbf{p}}\right),
$$

where $\mathbf{J}_{\mathbf{p}}$ and $\mathbf{M}_{\mathbf{p}}$ are current densities for the impressed electric and magnetic dipole sources, and Dirichlet boundary conditions are assumed with the tangential electric fields set to zero on the model domain boundary. Sometimes a scattered field formulation is desired because of accuracy considerations [2]. In this case we have

$\mathbf{S}=-i \omega \mu_{o}\left[\left(\sigma-\sigma_{\mathrm{p}}\right)+i \omega\left(\varepsilon-\varepsilon_{\mathrm{p}}\right)\right] \mathbf{E}_{\mathrm{p}}-i \omega \mu_{\mathrm{o}} \nabla_{\mathrm{h}} \mathrm{x}\left[\left(\mu-\mu_{\mathrm{o}}\right) / \mu \mathbf{H}_{\mathrm{p}}\right]$,

where $p$ designates background or primary values which are easy and fast to compute, such as a whole space. With a scattered field formulation the electric field, $\mathbf{E}$, in equation (1) is replaced by a scattered electric field, $\mathbf{E}_{\mathbf{s}}$, where we impose the boundary condition that tangential $\mathbf{E}_{\mathrm{s}}$ be zero on the boundary of the model domain. The total electric field is then given by the expression $\mathbf{E}_{t}=\mathbf{E}_{p}$ $+\mathbf{E}_{\mathbf{s}}$. In order to simulate natural source fields, $\mathbf{S}$ is set to zero everywhere, except at points where tangential
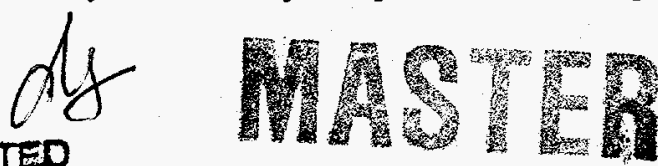


\section{DISCLAIMER}

This report was prepared as an account of work sponsored by an agency of the United States Government. Neither the United States Government nor any agency thereof, nor any of their employees, make any warranty, express or implied, or assumes any legal liability or responsibility for the accuracy, completeness, or usefulness of any information, apparatus, product, or process disclosed, or represents that its use would not infringe privately owned rights. Reference herein to any specific commercial product, process, or service by trade name, trademark, manufacturer, or otherwise does not necessarily constitute or imply its endorsement, recommendation, or favoring by the United States Government or any agency thereof. The views and opinions of authors expressed herein do not necessarily state or reflect those of the United States Government or any agency thereof. 


\section{DISCLAMMIXR}

Portions of this document may be illegible in electronic image products. Images are produced from the best available original document. 
electric-field boundary values are specified. These boundary values arise from vertically propagating plane waves in layered or $2 \mathrm{D}$ geologic media assigned at the boundaries of the $3 \mathrm{D}$ problem.

The modified differential operators in equation (1) are defined by

$$
\nabla_{\mathrm{e}}=\mathrm{i} 1 / e_{\mathrm{x}} \partial / \partial_{\mathrm{x}}+\mathrm{j} 1 / e_{y} \partial / \partial_{\mathrm{y}}+\mathrm{k} 1 / e_{z} \partial / \partial_{\mathrm{z}}
$$

and

$$
\nabla_{\mathrm{h}}=\mathbf{i} 1 / h_{x} \partial / \partial_{\mathrm{x}}+\mathbf{j} 1 / h_{y} \partial / \partial_{\mathrm{y}}+\mathbf{k} 1 / h_{z} \partial / \partial_{\mathrm{z}}
$$

where $e_{j}$ and $h_{j}$ for $j=x, y, z$ are complex coordinate stretching variables which stretch the $x, y$ and $z$ coordinates and define the PML absorbing boundary conditions originally developed by [5], but are in a form developed by [6] for ease of implementation.

When equation (1) is approximated with finite differences using the staggered grid due to [7], a linear system results in which the matrix is complex symmetric. This system can be efficiently solved iteratively using Krylov sub-space methods, including the quasi minimum residual (qmr) method. The reader is referred to [1] and [2] for the details on how these solvers are implemented. Once the electric fields are determined, the magnetic fields can be determined from Faraday's law, by numerically approximating the curl of the electric field at various nodal points and interpolating either the electric or magnetic field nodal values to the point of interest.

Even with the benefits of a staggered grid, which implicitly enforces the auxiliary divergence conditions on the current density,

$$
\nabla_{\mathrm{h}} \cdot\{(\sigma+i \omega \varepsilon) \mathbf{E}\}=\nabla_{\mathrm{h}} . \mathbf{S} / i \omega \mu_{\mathrm{o}},
$$

it is often necessary to explicitly enforce this condition through a static-divergence correction at frequencies approaching the static limit. The correction adds a term to the electric field such that equation (5) is identically satisfied and is alternated with a series of qmr iterations on equation (1). All the details on how to implement this correction procedure for either scattered and total fields can be found in [8], [9] and [10].

\section{MT Simulations}

In Figure 1, we demonstrate the 3D code's capability to simulate magnetotelluric fields which are useful in crustal investigations of the earth. The top part of the figure shows a conductive block, $2 \mathrm{~km}$ thick, residing in an earth with three layers. The 3D results are plotted at $1 \mathrm{~Hz}$ in terms of apparent resistivity and phase, given by the following formula and

$$
\begin{aligned}
& \rho^{\mathrm{TE}}=\left|\mathrm{E}_{y} / \mathrm{H}_{\mathrm{x}}\right|^{2} / \omega \mu_{0} \\
& \rho^{\mathrm{TM}}=\left|\mathrm{E}_{\mathrm{x}} / \mathrm{H}_{\mathrm{y}}\right|^{2} / \omega \mu_{0}
\end{aligned}
$$

$$
\begin{aligned}
& \theta^{\mathrm{TE}}=\tan ^{-1}\left[\operatorname{Im}\left(\mathrm{E}_{\mathrm{y}} / \mathrm{H}_{\mathrm{x}}\right) / \operatorname{Re}\left(\mathrm{E}_{\mathrm{y}} / \mathrm{H}_{\mathrm{x}}\right)\right] \\
& \theta^{\mathrm{TM}}=\tan ^{-1}\left[\operatorname{Im}\left(\mathrm{E}_{\mathrm{x}} / \mathrm{H}_{\mathrm{y}}\right) / \operatorname{Re}\left(\mathrm{E}_{\mathrm{x}} / \mathrm{H}_{\mathrm{y}}\right)\right] .
\end{aligned}
$$

The notation TE and TM stand for transverse electric and magnetic and denote responses produced by inducing electric and magnetic fields perpendicular to the strike direction of the model. For a half-space model, the apparent resistivity will equal that of the half space, while the phase will be 45 degrees. To verify the results, we have compared the 3D responses with two 2D codes; one based on finite elements [11] and the other on finite differences that we recently developed. In the $2 \mathrm{D}$ case the block model is assumed to possess infinite strike length, while in the $3 \mathrm{D}$ case it is $80 \mathrm{~km}$. Comparisons between the different codes are quite good. It must be noted that to obtain accurate 3D results a static divergence correction, as discussed above, was required for this simulation.
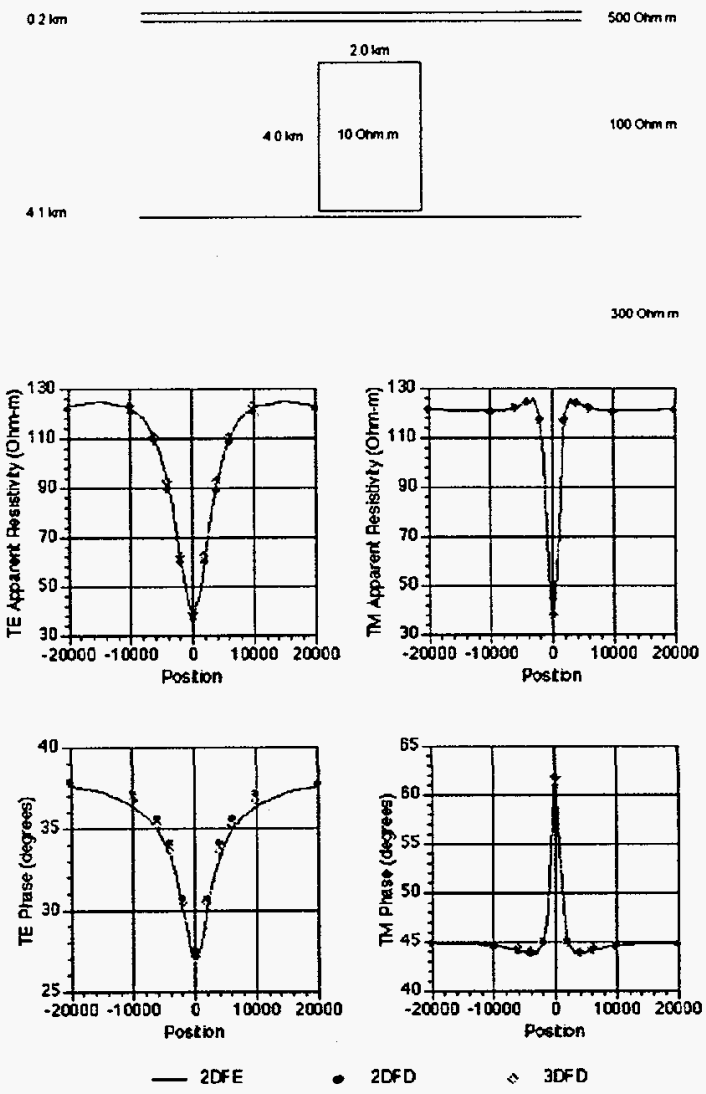

Figure 1. Comparison between 3D and 2D codes for a MT simulation at $1 \mathrm{~Hz}$ at the earth's surface. 
The Need for PML Boundary Conditions

[1] has shown the need of the PML absorbing boundary conditions when simulating propagating fields in the frequency domain. To summarize the results, the complex stretching parameters are assigned a value of 1 $+a-i b$. On the internal portions of the mesh, $a=b=0$ such that the modified Helmholtz equation reduces to the normal form. Near edges of the mesh $e_{i}$ and $h_{i}$ are allowed to vary over several cells, but only in the direction that is perpendicular to the boundary. For example, along the $+z$ boundary $e_{x}=e_{y}=h_{x}=h_{y}=1$ and only $e_{z}=h_{z}$ are allowed values of $a$ and $b$ that differ from zero. Some guidelines for selecting the PML stretching coordinates can be found in [1].

Figure 2 shows a simulation where the horizontal and vertical magnetic field arising from a vertical magnetic dipole and calculated on a $120 \times 120 \times$ 120 mesh for the three layer model is indicated in panel a). Resistivities of the layers are $1373,76,1000 \Omega$.m and 6,41 and 1 are their dielectric properties relative to free space. The thickness of each layer is 1.1 and 0.9 meters. The 3D results and have been plotted against the ID solution based on Sommerfield integrals. It is immediately evident in panel b) that the 3D solution without the PML boundary condition begins to break down at about $15 \mathrm{MHz}$, and we can assume that this is due to reflections due to the mesh boundaries contaminating the solution. Doubling the size of the cells along the mesh boundaries, that is using real grid stretching, does not help matters. Panel c) shows that poor results occur when a real stretching parameter $a=$ 1.0 is employed along 25 cells of each boundary. However, when complex grid stretching is employed the results are much better. Panel d) shows results that when a stretching parameter of $b=-0.6$ is employed along 25 cells of each boundary, the fields calculated with the $3 \mathrm{D}$ solution match those of the ID solution almost exactly. In panel e) we demonstrate how absorbing boundaries can be applied to shrink the size of the mesh. In this case a $72 \times 72 \times 72$ mesh was used along with $b=-2.0$ applied for 10 cells along each boundary. Notice that both the 3D and 1D calculations coincide. This example fully illustrates the utility of the PML boundary conditions as they not only allow one to accurately simulate wave propagation, but also allow the mesh size to be significantly reduced and results in much smaller run times.

\section{ACKNOWLEDGMENTS}

This work was performed at Sandia National Laboratories, which is operated for the United States Department of Energy (DOE). Funding for the work was
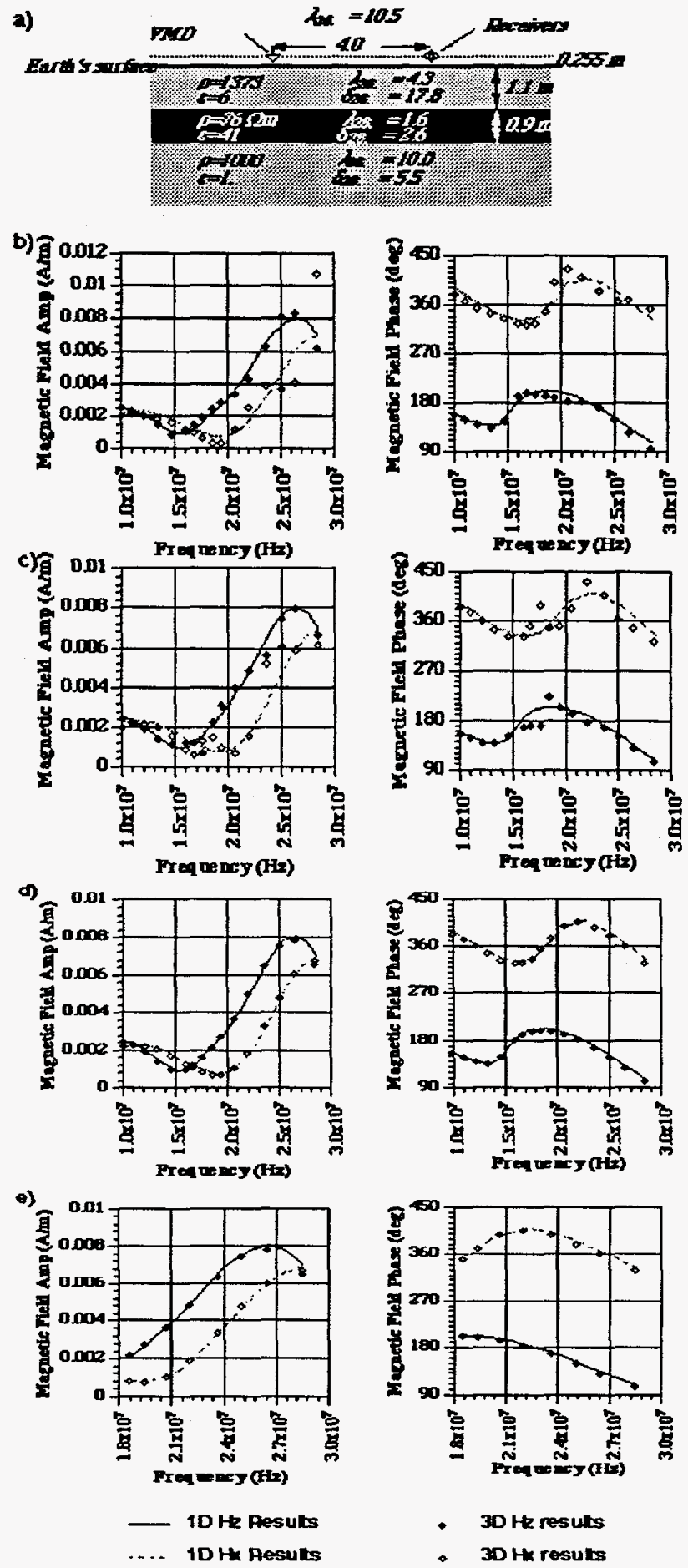

- 30 He resuls

- 30 H results

Figure 2. ID model used to verify the need for PML absorbing boundary conditions. Note that the magnetic permeability has been set to that of free space. At the top of the figure we also have included wavelengths and skin depths in the respective layers. See text for panel explanation. 
provided by DOE's office of Basic Energy Sciences, Division of Engineering and Geoscience, under contract DE-AC04-94AL85000.

\section{REFERENCES}

[1] Alumbaugh D. L., Newman G. A., Prevost L., and Shadid, J. N., 1996, Three-dimensional wideband electromagnetic modeling on massively parallel computers: Radio Science, 31, 1-23.

[2] Newman G. A., and Alumbaugh D. L., 1995, Frequency-domain modeling of airborne electromagnetic responses using staggered finite differences: Geophysical Prospecting, 43, 1021-1042.

[3] Newman G. A., and Alumbaugh D. L., 1997, Threedimensional massively parallel electromagnetic inversion - I. Theory: Geophysical Journal International, 128, 345354.

[4] Alumbaugh D. L., and Newman G. A., 1997, Threedimensional electromagnetic inversion - II. Analysis of a crosswell electromagnetic experiment: Geophysical Journal International, 128, 355-363.

[5] Berrenger J., 1993, A perfectly matched layer for the absorption of electromagnetic waves: Journal of Computational Physics, 114, 185-200.

[6] Chew W. C., and Weedon W. H., 1994, A 3D perfectly matched medium from modified Maxwell's equations with stretched coordinates: Microwave Optical Technology Letters, 7, 599-604.

[7] Yee K. S., 1966, Numerical solution of initial boundary problems involving Maxwell's equations in isotropic media: IEEE Transactions on Antennas and Propagation, AP-14, 302-309.

[8] Newman G. A., and Alumbaugh, D. L., 1996, Electromagnetic modeling of subsurface 3D structures: Presented at the International Geoscience and Remote Sensing Symposium (IGARSS), Omaha, Nebraska.

[9]Alumbaugh D. L., and Newman G. A., 1996, Electromagnetic modeling of perfect conductors in an arbitrary host, $66^{\text {th }}$ Society of Exploration Geophysicists Annual Meeting, Extended Abstracts, 978-981, Tulsa OK.

[10] Smith T., Conservative modeling of 3D electromagnetic fields; Part I: Properties and error analysis: Geophysics, 61, 1308-1318.
[11]Wannamaker P. E., Stodt J. A., and Rijo L., 1987, A stable finite element solution for two-dimensional magnetotelluric modeling: Geophysical Journal Royal Astronomical Society: 88, 277-296 